\title{
THE EFFECT OF VARIATIONS IN CULTURE CONDITIONS ON THE MOTILITY OF HAMSTER SPERMATOZOA
}

\author{
B. D. BAVISTER* \\ Physiological Laboratory, Cambridge \\ (Received 3rd October 1973)
}

\begin{abstract}
Summary. The motility of hamster epididymal spermatozoa has been studied in modified Tyrode's solution; a high level of motility was maintained when bovine serum albumin, used in previous studies, was replaced by polyvinyl pyrrolidone, and when no macromolecule was included. In these culture media, it was found that sperm motility and survival were considerably enhanced if the osmotic pressure was lowered by $20 \%$ from the usual level of 290 mosmol. The reduced osmotic pressure caused leakage of a motility-stimulating substance from spermatozoa; the need to lower the osmotic pressure for optimum sperm motility could be eliminated by adding an extract of spermatozoa to the culture medium. With this culture system, spermatozoa could be maintained in a high state of motility for several hours, and the 'sperm dilution effect' was substantially reduced.
\end{abstract}

\section{INTRODUCTION}

It was previously reported that epididymal spermatozoa from the golden hamster can be capacitated by incubating them in a culture medium (Bavister, 1971,1973 ). This study of the behaviour of hamster spermatozoa in culture media was restricted by the practical difficulties involved in finding conditions suitable for maintaining vigorous sperm motility for long enough to enable capacitation to occur ( $3 \frac{1}{2}$ to $5 \mathrm{hr}$ ). The main problem was that of sperm concentration: at concentrations of approximately $10^{8} / \mathrm{ml}$, the quality of motility often deteriorated considerably within a few hours, perhaps due to an accumulation of metabolites. The performance of spermatozoa was not improved by lowering the sperm concentration, for hamster spermatozoa show the 'sperm dilution effect' (discussed by Mann, 1964) to a remarkable extent. A compromise was reached by incorporating the cauda epididymidis itself in the sperm suspension, which considerably extended the length of time for which spermatozoa showed vigorous motility, and by incubating sperm suspensions at 25 to $30^{\circ} \mathrm{C}$ rather than $37^{\circ} \mathrm{C}$. The culture medium used for this work was a modified Tyrode's solution containing sodium pyruvate and bovine serum albumin (BSA).

The present study was undertaken in order to improve the culture environ-

\footnotetext{
* Present address: School of Medicine, Department of Anatomy, 1960 East-West Road, Honolulu, Hawaii 96822, U.S.A.
} 
ment for survival of hamster spermatozoa, with a view to designing a simplified culture system in which spermatozoa would retain a high level of motility after incubation for several hours, and thereby facilitate investigation of the mechanism of capacitation in culture media. Ideally, the culture medium should be completely synthetic; it has been suggested (Yanagimachi, 1970a; Miyamoto \& Chang, 1973) that BSA may play an active rôle in capacitation in vitro. Moreover, the suitability of BSA for culture work, especially fertilization in vitro, varies considerably from one batch to another (Bavister, 1971).

In a preliminary investigation, it was found that hamster spermatozoa remained motile substantially longer when the osmotic pressure of the medium was reduced by $20 \%$. Accordingly, the first part of the present work is concerned with the effect of osmotic pressure on sperm motility, and with the substitution of BSA by a synthetic polymer, polyvinyl pyrrolidone (PVP).

\section{MATERIALS AND METHODS}

\section{Culture media}

The basic culture medium used in this study was Tyrode's solution supplemented as follows: Tyrode's solution, $100 \mathrm{ml}$; sodium pyruvate, $1 \mathrm{ml}$ of a 20 mM solution; sodium penicillin G, 10,000 i.u.; sodium bicarbonate, $4.5 \mathrm{ml}$ of $1.4 \%$ solution. All inorganic chemicals were analytical grade (B.D.H. 'Analar').

This basic culture medium served as a control for the effect of macromolecules on sperm motility: either BSA or PVP (May \& Baker, mol. wt given as 40,000) was added at $1 \% \mathrm{w} / \mathrm{v}$ concentration. To remove toxic material of low molecular weight, PVP was dialysed against water for 3 days, as suggested by AshwoodSmith \& Warby (1971), followed by freeze-drying. The osmotic pressure of the basic culture medium was $290 \mathrm{mosmol}$ : this value was not increased by more than 1 or 2 mosmol by addition of macromolecules in the concentrations stated.

Media with an osmotic pressure of 230 mosmol were obtained either by diluting the basic culture medium with distilled water, or by lowering the $\mathrm{NaCl}$ and KCI content of the Tyrode's solution. In practice, no difference was found in terms of sperm motility between these two methods, and the former was routinely employed. All culture media were passed through a $0.22 \mu \mathrm{m}$ 'millipore' filter and stored at $+4^{\circ} \mathrm{C}$ until needed; media more than 1 week old were discarded.

\section{Insemination of culture droplets}

Several 100- $\mu$ l drops of each culture medium were placed under paraffin oil (light grade, Boots Pure Drug Co.) in plastic culture dishes (Falcon Plastics), and equilibrated overnight with $5 \% \mathrm{CO}_{2}$ in air in a continuous-flow gas incubator at $37^{\circ} \mathrm{C}$; the final $\mathrm{pH}$ was $7 \cdot 4$ to $7 \cdot 6$. Next morning, spermatozoa were obtained from 3- to 4-month-old golden hamsters by severing the tubule of the excised cauda epididymidis at several points and allowing the contents to exude. This procedure was performed under paraffin oil in a plastic tissue culture dish at room temperature. Between $15 \mathrm{~min}$ and $1 \mathrm{hr}$ after releasing the epididymal contents, aliquots were taken up in a siliconed 2- $\mu$ l 'Microcap' pipette (Drummond) that had previously been subdivided in $0 \cdot 1-\mu$ l steps with glass- 
marking ink, and used to inseminate the drops of culture media, which were then reincubated.

The sperm concentration of fresh epididymal contents was determined with a Hawksley haemocytometer, and found to vary between 1.32 and $2.58 \times 10^{9} / \mathrm{ml}$ in fifteen males tested (mean $1.98 \pm$ S.E. $0.11 \times 10^{9} / \mathrm{ml}$ ). A volume of epididymal contents from each male was adjusted as necessary with basic culture medium to produce a suspension containing $10^{9}$ spermatozoa $/ \mathrm{ml}$. Appropriate volumes of this suspension were deposited in the drops of culture medium to produce desired concentrations of $10^{7}, 2 \times 10^{6}$ and $4 \times 10^{5}$ spermatozoa $/ \mathrm{ml}$. Ten drops of each sperm concentration were checked with the haemocytometer to assess the accuracy of the insemination technique; the mean and S.E.M. of these determinations were $1.15 \pm 0.08 \times 10^{7}, 1.91 \pm 0.15 \times 10^{6}$ and $3.83 \pm 0.37 \times 10^{5}$ per ml, respectively.

\section{Motility scoring}

At intervals, each dish was withdrawn from the incubator and the drops were examined under the dissecting microscope to estimate the degree of sperm motility. This was assessed in two ways: (1) the percentage of spermatozoa showing movement was estimated under $\times 25$ magnification; (2) the average degree of motility was estimated on a 0 to 5 scale, on which Grades 1 to 4 were arbitrary divisions on a scale of increasing vigour and progressive motility, and Grade 5 was reserved for spermatozoa showing a characteristic frenzied 'whip-lash' action of high amplitude, together with frequent changes in the direction of swimming. This type of motility can readily be distinguished under $\times 50$ magnification from vigorous but low amplitude tail movements of Grade 4 , and is also exhibited by hamster spermatozoa that have been incubated for several hours with cumulus masses.

In order to obtain a useful index of sperm motility, the proportion of spermatozoa in each drop showing motility $(0$ to $80 \%)$ was multiplied by the square of the value for degree of motility (since the latter parameter was regarded as being more significant for capacitation studies).

While subjective methods of assessing sperm motility are not ideal, they are preferable, in my experience, to methods involving transfer of aliquots of sperm suspensions to a microscope slide for more objective measurement, since this simple procedure often results in a considerable decrease in motility, especially after spermatozoa have been incubated for some hours in culture media. The error inherent in the subjective scoring method used here was reduced by assigning randomized numbers to the treatments in the evening when setting up the experiment, so that the drops of different culture media could not be distinguished next day when they were inseminated.

\section{Preparation of sperm extracts and testing}

Hamster epididymal contents were collected as previously described, allowing several hours for sufficient fluid to accumulate in the culture dish. Care was taken to minimize tissue damage and contamination of the spermatozoa with blood. Approximately $150 \mu \mathrm{l}$ pooled epididymal contents from several hamsters were diluted with $0.5 \mathrm{ml}$ basic culture medium, buffered with TES and HEPES, 
each at $15 \mathrm{~mm}$, to $\mathrm{pH} 7.4$; the osmotic pressure of this medium was 300 mosmol. When the spermatozoa had dispersed in the medium, the suspension was centrifuged at room temperature at $1200 \mathrm{~g}$ for $5 \mathrm{~min}$, after which the supernatant of diluted epididymal contents was removed. The pellet of spermatozoa was resuspended in $10 \mathrm{ml}$ of the same medium, with gentle suction through a Pasteur pipette. About $50 \%$ of the spermatozoa were still motile after this treatment, and most of the motile spermatozoa had intact acrosomes (as judged under phase-contrast microscopy); however, many of the immotile spermatozoa had undergone an acrosome reaction. The suspension was centrifuged at $800 \mathrm{~g}$ for $5 \mathrm{~min}$ at room temperature; all of the supernatant and the upper layer of the pellet was removed. The remaining sperm pellet was resuspended as before in $1 \mathrm{ml}$ culture medium: about half of the motile spermatozoa still had intact acrosomes. This suspension was plunged into liquid nitrogen for a few minutes, then allowed to thaw at room temperature. This treatment very effectively disrupted the remaining acrosomes and released their contents. The preparation was then centrifuged at $2250 \mathrm{~g}$ for $10 \mathrm{~min}$, and the supernatant was collected and passed through a millipore filter. Freezing in liquid nitrogen, followed by thawing, has been described as a very convenient method for obtaining a preparation of acrosomal contents from human spermatozoa (Pedersen, 1972).

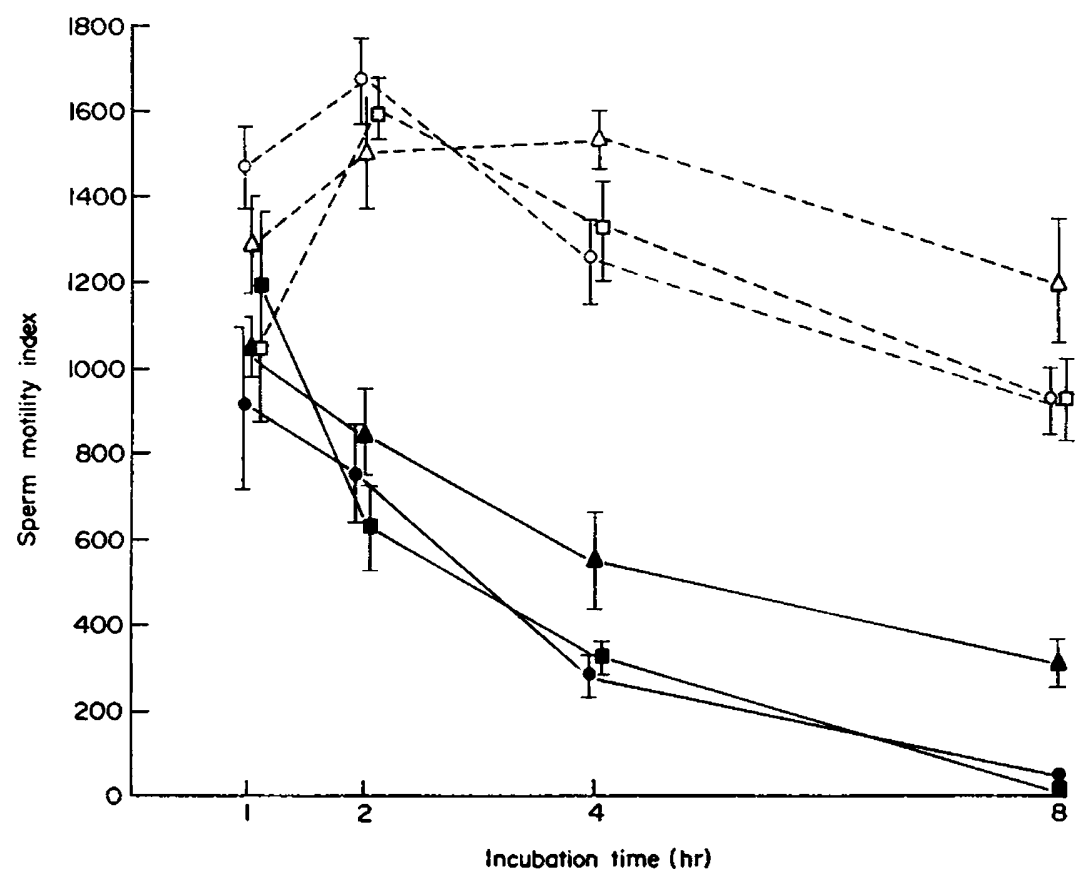

TEXT-FIG. 1. Motility of hamster spermatozoa in synthetic and bovine serum albumincontaining culture media, and effect of osmotic pressure. Sperm concentration is approximately $10^{7} / \mathrm{ml}$. Each point is the mean of six observations, with spermatozoa from four males. Vertical bars represent S.E.M. The media used were as follows: basic medium + bovine serum albumin, $\Delta(290 \mathrm{mosmol})$ and $\Delta(230 \mathrm{mosmol})$; basic medium + polyvinyl pyrrolidone, $\bullet(290 \mathrm{mosmol})$ and $\mathrm{O}(230 \mathrm{mosmol})$; basic medium only, (290 mosmol) and $\square(230 \mathrm{mosmol})$. 
In the present case, however, spermatozoa were not treated with glycerine before freezing, and it is likely that the extracts contained material from other regions of the spermatozoa as well as from the acrosomes.

Sperm extracts were prepared in this way on three separate occasions, and tested for their ability to maintain the motility of hamster epididymal spermatozoa at 290 mosmol in a defined culture medium (basic medium $+1 \%$ PVP). Each of the extracts was diluted 1:9 in the culture medium, and 100- $\mu$ l drops were prepared and equilibrated overnight under oil with $5 \% \mathrm{CO}_{2}$ in air. Drops of defined culture medium alone were included as controls. Next day, the drops were inseminated as before with fresh epididymal spermatozoa. Spermatozoa from four hamsters were tested for motility in this way.

\section{RESULTS}

When hamster epididymal spermatozoa were incubated in culture media at 290 mosmol, the index of motility declined steadily with time (Text-fig. 1) but at 230 mosmol, there was a striking enhancement of motility for the first $2 \mathrm{hr}$, after which the motility index declined at about the same rate as in the controls. No difference was found in results between the medium with PVP and the basic culture medium, but the inclusion of BSA in the medium produced a slight enhancement of motility at both osmotic pressures at the 4- and 8-hr time-intervals. After incubation for 4 and $8 \mathrm{hr}$, combined results at $230 \mathrm{mosmol}$ were significantly different from those at 290 mosmol $(P<0.001$, by analysis of variance). The level of significance between the individual media, calculated by the $t$ test, was as follows:

Incubation for:

BSA-medium versus PVP-medium PVP-medium versus basic medium basic medium versus BSA-medium

$\begin{array}{cc}4 \mathrm{hr} & 8 \mathrm{hr} \\ P<0.01 & P<0.01 \\ \text { N.S. } & \text { N.S. } \\ P<0.05 & P<0.01\end{array}$

There was no interaction between culture media and osmotic pressure.

Since the appearance of Grade 5 motility may be associated with capacitation, its frequency was recorded (Table 1 ). This type of motility was nearly always observed in media at 230 mosmol, sometimes persisting for $8 \mathrm{hr}$, in contrast to the results at 290 mosmol.

Since there was no obvious explanation of the effect of osmotic pressure, sperm motility at the beginning of the incubation period was examined. Drops of culture media were inseminated as before with epididymal spermatozoa at $10^{7} / \mathrm{ml}$, and examined after $5 \mathrm{~min}$. Usually, hamster spermatozoa disperse rapidly in culture media, with vigorous motility but consistently at $230 \mathrm{mosmol}$ in all three culture media, a proportion (estimated at 10 to $20 \%$ ) of the spermatozoa failed to become motile within 5 min whereas at 290 mosmol, few immotile spermatozoa were found within this time. The spermatozoa that failed to become motile at 230 mosmol within 5 min did not subsequently recover and, when examined under the phase-contrast microscope, all of them were found to have undergone acrosomal lysis, presumably caused by some adverse effect of low osmotic pressure. The possibility arose that the acrosomal contents released 
by these spermatozoa at the beginning of incubation in some way maintained the motility of the surviving spermatozoa.

A suspension of epididymal spermatozoa was prepared and the cells were inactivated by standing the tube in hot water for a few minutes. The suspension was centrifuged, and the supernatant was added to culture medium at 290 mosmol containing PVP, which was then inseminated with fresh epididymal spermatozoa at $10^{7} / \mathrm{ml}$. The index of motility in this preparation was similar to that previously observed at 230 mosmol.

Sperm motility was next examined in basic culture medium with PVP at 290 mosmol, to which an extract of epididymal spermatozoa had been added. At sperm concentrations of approximately $10^{7} / \mathrm{ml}$ (Text-fig. 2a), the index of sperm motility in the presence of sperm extracts reached a similar value, after incubation for $2 \mathrm{hr}$, to that obtained with PVP-medium at 230 mosmol (Text-fig. 1); after $2 \mathrm{hr}$, the motility decreased rapidly, falling to near control values by $8 \mathrm{hr}$.

Table 1. Effect of osmotic pressure on the motility of hamster spermatozoa: frequency of scoring Grade 5 motility

\begin{tabular}{|c|c|c|c|c|c|}
\hline \multirow{2}{*}{$\begin{array}{l}\text { Culture medium } \\
\text { containing }\end{array}$} & \multirow{2}{*}{$\begin{array}{c}\text { Osmotic } \\
\text { pressure } \\
\text { (mosmol) }\end{array}$} & \multicolumn{4}{|c|}{ Incubation period $(h r)$} \\
\hline & & 1 & 2 & & 8 \\
\hline BSA & 290 & 4 & 2 & 1 & 0 \\
\hline PVP & 290 & 1 & 0 & 0 & 0 \\
\hline No macromolecule & 290 & 3 & 0 & 0 & 0 \\
\hline BSA & 230 & 6 & 6 & 6 & 3 \\
\hline PVP & 230 & 6 & 6 & 2 & 0 \\
\hline No macromolecule & 230 & 5 & 6 & 3 & 1 \\
\hline
\end{tabular}

$\mathrm{BSA}=$ bovine serum albumin; $\mathrm{PVP}=$ polyvinyl pyrrolidone. Figures given are the number of duplicate experiments (out of six) in which Grade 5 sperm motility (see 'Materials and Methods') was observed. Sperm concentration $10^{7} / \mathrm{ml}$.

A similar effect of the sperm extracts was seen with $2 \times 10^{6}$ spermatozoa $/ \mathrm{ml}$, the peak of the motility index being reached at $4 \mathrm{hr}$ (Text-fig. 2b). At the lowest sperm concentration tested $\left(4 \times 10^{5}\right.$ spermatozoa $\left./ \mathrm{ml}\right)$, there was still considerable enhancement of motility by sperm extracts (Text-fig. 2c).

The frequency of scoring Grade 5 motility in spermatozoa incubated at $10^{7} / \mathrm{ml}$ with sperm extracts is recorded in Table 2. Two of the three extracts used always produced this type of motility following incubation for $2 \mathrm{hr}$, but the third extract failed to do so. Grade 5 motility was also seen at the two lower sperm concentrations tested, but only occasionally and inconsistently, so these results are not given.

A comparison of the control values in Text-figs 2(a) to 2(c) gives an indication of the extent of the sperm dilution effect with hamster spermatozoa over a 25-fold range of concentration. The motility index values obtained in the presence of the sperm extracts show that the sperm dilution effect was substantially reduced by these extracts. 


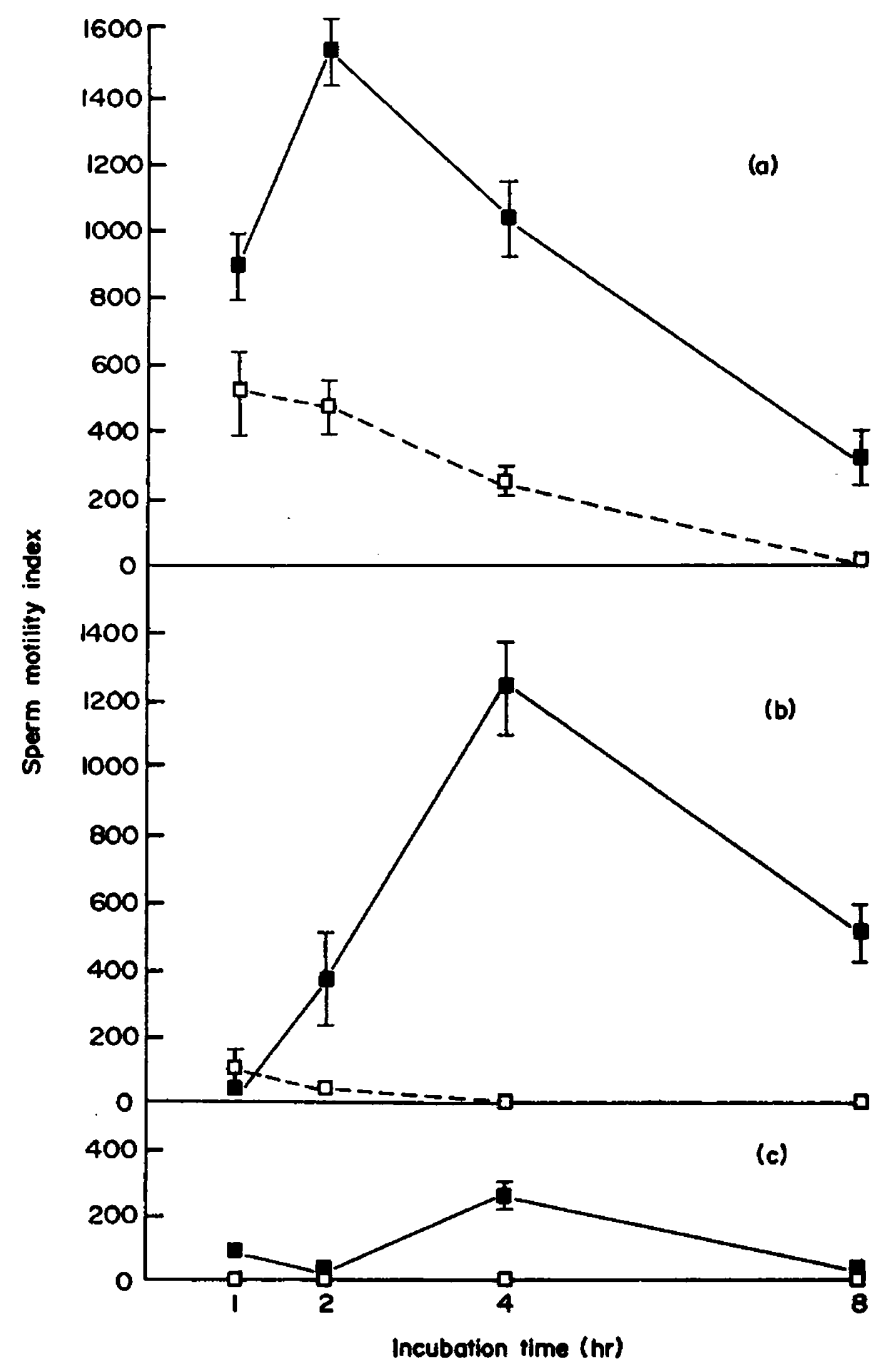

TEXT-FIG. 2. Motility of hamster spermatozoa in synthetic culture medium. Sperm concentrations: (a) $10^{7} / \mathrm{ml}$, (b) $2 \times 10^{6} / \mathrm{ml}$, (c) $4 \times 10^{5} / \mathrm{ml}$. $\mathrm{m}$, Basic culture medium containing sperm extract: each point is the mean of twelve observations, with three sperm extract preparations; $\square$, basic culture medium only (controls): each point is the mean of eight observations. Vertical bars represent S.E.M. 
Table 2. Motility of hamster spermatozoa incubated with sperm extracts: frequency of scoring Grade 5 motility

\begin{tabular}{c|cccc}
\hline \multirow{2}{*}{ Extract no. } & \multicolumn{4}{|c}{ Incubation } \\
& 1 & 2 & 4 & 8 \\
\hline 1 & 1 & 4 & 2 & 0 \\
2 & 2 & 4 & 1 & 0 \\
3 & 0 & 0 & 0 & 0 \\
\hline
\end{tabular}

Values given are the number of duplicate experiments (out of four) in which Grade 5 motility (see 'Materials and Methods') was scored. Sperm concentration $10^{7} / \mathrm{ml}$.

\section{DISCUSSION}

The rapid decline in motility exhibited by hamster spermatozoa at 290 mosmol, with a roughly 200 -fold dilution of epididymal contents (Text-fig. 1), illustrates their remarkable sensitivity to the sperm dilution effect; undiluted epididymal spermatozoa can be stored under oil at room temperature for 12 to $24 \mathrm{hr}$, without serious loss of motility when ultimately added to culture medium (B. D. Bavister, unpublished observation). It is a little surprising that BSA did not produce a larger rise in the motility index compared with the defined media (Text-fig. 1), in view of its supposed protective effect on cells, especially spermatozoa, in culture (discussed by Mann, 1964). Although the epididymal fluid contained in these sperm suspensions is present in very small amounts, it may have exerted some protective action on the spermatozoa. The improvement in motility in the presence of BSA (Text-fig. 1) was statistically significant, but rather small, while the inclusion of BSA in the culture medium prolonged the period during which the highest level of sperm motility was seen (Table 1). In spite of the general improvement in sperm motility with BSA, one of the aims of the present investigation was to demonstrate that BSA is not essential for the maintenance of good motility, and the results with defined culture media (Text-fig. 1, Table 1) approached those, and were sometimes similar to, results obtained with BSA. In view of this, subsequent experiments were carried out with a protein-free culture medium. Although no difference in sperm motility was found in the present work between media with and without PVP, the results of several preliminary experiments with low sperm concentration $\left(10^{5}\right.$ to $10^{6} / \mathrm{ml}$ ) had sometimes demonstrated a beneficial effect of PVP (B. D. Bavister, unpublished observations). For this reason, PVP was included in the defined culture medium used subsequently.

The increased motility of hamster spermatozoa in culture media with an osmotic pressure of 230 mosmol (Text-fig. 1) may be explained by the release of a motility-stimulating substance from a proportion of the spermatozoa introduced into the culture medium. These spermatozoa, presumably the most 
sensitive to osmotic shock, undergo an almost immediate acrosome reaction, and are immotile. The motility-stimulating activity can be extracted from washed spermatozoa by freezing and thawing, and may come from the acrosome, although the possibility that it is derived from other regions of the cell cannot be ruled out at present. The nature of the substance responsible for the stimulation of motility is not yet known. The high level of motility induced by incubation of spermatozoa at $230 \mathrm{mosmol}$, or with sperm extracts at $290 \mathrm{mosmol}$, lasts only for 2 to $4 \mathrm{hr}$, after which motility falls rapidly towards control values. One explanation for this behaviour is that the active substance is a metabolite, which is used up after 2 to $4 \mathrm{hr}$. The sperm dilution effect could be explained in terms of a sperm motility-stimulating substance located within the spermatozoon itself: if this substance were capable of slowly diffusing out of the spermatozoon, then increasing the ratio of diluent : sperm cells would progressively lower the concentration of this substance within the spermatozoa.

Lardy, Ghosh \& Plaut (1949) extracted a 'metabolic regulator' from bull epididymal spermatozoa by alkaline hydrolysis. This substance, which also leaked out of spermatozoa during storage, apparently uncoupled oxidative phosphorylation. If the factor derived from hamster spermatozoa acts in a similar way, it might explain why high motility was only maintained for 2 to $4 \mathrm{hr}$, followed by a rapid fall, possibly as internal energy stores were depleted.

The existence of a substance that stimulated the motility of rabbit spermatozoa, and was able to counteract the dilution effect, was demonstrated by Emmens \& Swyer (1948). They found this activity in cell-free supernatants of rabbit semen, and the most effective samples were those obtained from suspensions of spermatozoa that had been left overnight before centrifuging, indicating that the active agent originated within the spermatozoa themselves. It seems likely that the agent discovered by Emmens \& Swyer is similar to that described in the present work, which also substantially reduces the dilution effect.

The type of motility frequently exhibited by spermatozoa incubated at 230 mosmol (Table 1), or 290 mosmol in the presence of sperm extract (Table 2), namely, a flagellar wave-form of very high amplitude (Grade 5 motility), is remarkably similar to that described by Yanagimachi (1970b) in his elegant experiments involving microcinematography of hamster spermatozoa. He demonstrated that this type of motility was exhibited by spermatozoa only after capacitation. While it has not yet been shown that hamster spermatozoa incubated with sperm extracts become capacitated, it is interesting that this type of motility can now be obtained with sperm extracts, whereas previously it has only been achieved, in the hamster, in the presence of the fluid component of the cumulus mass (Yanagimachi, 1969a), with various blood sera or with heterologous follicular fluid (Yanagimachi, 1969b, 1970a, b). Grade 5 motility was obtained consistently with sperm extracts 1 and 2, but not with extract 3 ; this discrepancy suggests that the conditions for extracting the motility-stimulating substance from spermatozoa have not been sufficiently elucidated. Barros, Berrios \& Herrera (1973) have obtained similar motility of guinea-pig spermatozoa by incubating them in a defined culture medium devoid of macromolecules. Spermatozoa treated in this way were able to penetrate zona-free hamster eggs, whereas untreated spermatozoa were not. Yanagimachi (1972) 
has also noted this type of motility in guinea-pig spermatozoa during incubation in culture medium.

It is clear that expression of the type of motility described above in hamster and in guinea-pig spermatozoa is linked with the achievement of capacitation; the present work, and that of Barros et al. (1973), show that BSA is not essential for attainment of such motility in these species. Moreover, in the experiments of Barros et al. (1973), guinea-pig spermatozoa were apparently capacitated in the absence of BSA. It seems unlikely therefore that BSA plays a direct rôle in capacitation in vitro.

Experiments are now in progress to discover if hamster spermatozoa incubated with sperm extracts undergo capacitation, and to ascertain the nature of the motility-stimulating activity contained in these extracts, and whether it is related to that in blood sera and follicular fluid.

\section{ACKNOWLEDGMENTS}

I am grateful to Miss D. Suckling for technical assistance during this work, to Mr I. Walters for discussing the analysis of results, and to the Ford Foundation for financial support of this investigation.

\section{REFERENCES}

Ashwood-Smith, M. \& WARBy, C. (1971) Studies on the molecular weight and cryoprotective properties of polyvinylpyrrolidone and dextran with bacteria and erythrocytes. Cryobiology, 8, 453.

Barros, G., Berrios, M. \& Herrera, E. (1973) Capacitation in vitro of guinea-pig spermatozoa in a saline solution. F. Reprod. Fert. 34, 547.

BAvister, B.D. (1971) A study of in vitro fertilization and capacitation in the hamster. Ph.D. thesis, Cambridge University.

Bavister, B. D. (1973) Capacitation of golden hamster spermatozoa during incubation in culture medium. 7. Reprod. Fert. 35, 161.

Emmens, C. W. \& SWYer, G. I. M. (1948) Observations on the motility of rabbit spermatozoa in dilute suspension. J. gen. Physiol. 32, 121.

Lardy, H. A., Ghosh, D. \& Plaut, G. W. E. (1949) A metabolic regulator in mammalian spermatozoa. Science, N.Y. 109, 365.

Mann, T. (1964) The Biochemistry of Semen and of the Male Reproductive Tract, pp. 339-350. Methuen, London.

Miyamoto, H. \& Chang, M. C. (1973) The importance of serum albumin and metabolic intermediates for capacitation of spermatozoa and fertilization of mouse eggs in vitro. F. Reprod. Fert. 32, 193.

Pedersen, H. (1972) The acrosome of the human spermatozoon: a new method for its extraction and an analysis of its trypsin-like enzyme activity. J. Reprod. Fert. $31,99$.

YANAGIMACHI, R. (1969a) In vitro capacitation of hamster spermatozoa by follicular fluid. F. Reprod. Fert. 18, 275.

YANAGIMACHI, R. (1969b) In vitro acrosome reaction and capacitation of golden hamster spermatozoa by bovine follicular fluid and its fractions. F. exp. Zool. 170, 269.

YANAGIMACHI, R. (1970a) In vitro capacitation of golden hamster spermatozoa by homologous and heterologous blood sera. Biol. Reprod. 3, 147.

YANAGIMAGH, R. (1970b) The movement of golden hamster spermatozoa before and after capacitation. J. Reprod. Fert. 23, 193.

YanagimachI, R. (1972) Fertilization of guinea-pig eggs in vilro. Anat. Rec. 174, 9. 